Ann. Biol. anim. Bioch. Biophys., 1977, 17 (3 B), 473-476.

\title{
Existence de neurones sensibles à la prolactine dans les régions préoptiques et septales du Cobaye : étude par micro-iontopho- rèse.
}

par P. POULAIN, B. CARETTE

Laboratoire d'Histologie, Facultè de Médecine, place de Verdun, 59045 Lille Cedex

Summary. Prolactin-sensitive neurons in the preoptic and septal areas of the guinea-pig studied with the microiontophoresis technique.

With the microiontophoresis technique we determine the direct effect of prolactin on the unit activity of preoptic and septal neurons of the guinea-pig. Twenty-seven cells show no chonge in firing rate after application of prolactin. The remaining 36 showing hormone response are divided into two types depending on the time course of the effect. Unresponsive cells and neurons with rapid effects in onset and recovery ( 3 activated, 6 inhibited) are scattered throughout the septum and preoptic areas. The long-lasting excitatory effect $(27$ cells) of prolactin appears predominant in the preoptic area. No peculiar time-course of prolactin effects is observed on neurons which project directly to the median eminence. This study shows the presence of prolactin-sensitive neurons in the preoptic and septol areas of the guinea-pig.

\section{Introduction.}

Différentes études utilisant les techniques de stimulation électrique ou électrochimique permettent d'inclure l'aire préoptique (APO) dans les régions diencéphaliques jouant un rôle de contrôle de la sécrétion de prolactine (PRL). Cependant l'état contradictoire des résultats ne permet pas de préciser le rôle particulier de cette aire : inhibition (Clemens et al., 1971 b; Kawakami et al., 1973b) et activation (Wuttke et Meites, 1972 ; Kawakami et al., 1973a ; Malven, 1975) de la libération de PRL sont obtenues après l'application de chocs électriques dans la région préoptique. L'existence de neurones sensibles à la PRL dans cette région a été suggérée par Clemens ef al. (1971 a) qui montrèrent les effets inhibiteurs et activateurs d'une injection intraveineuse de PRL sur l'activité électrique des neurones de l'APO.

Pour tenter d'étudier le comportement de ces neurones soumis cette fois à l'application directe de $\mathrm{PRL}$, nous avons utilisé la technique de micro-iontophorèse sur les cellules rencontrées dans les aires préoptiques et septales du cobaye. La possibilité de déclencher l'activité antidromique de certaines de ces cellules en appliquant un choc électrique à l'éminence médiane a prouvé leur relation directe avec l'aire stimulée (Poulain et Partouche, 1973). 


\section{Techniques expérimentales.}

L'étude porte sur sept cobayes femelles de $400 \mathrm{~g}$, anesthésiés par un mélange nembutal-uréthane et placés sous respiration artificielle après une injection de flaxédil.

Une électrode de stimulation de type bipolaire est implantée dans l'éminence médiane et transmet des chocs de $1 \mathrm{~ms}$. d'intensité comprise entre 0,5 et $1,5 \mathrm{~m}$.

Les microélectrodes à 4 canaux de verre soudés ont un diamètre de 3 à $4 \mu$ à la pointe. Les potentiels d'action sont recueillis en extracellulaire à partir d'un des canaux empli d'une solution de $\mathrm{NaCl} 2 \mathrm{M}$. Après amplification, les potentiels d'action sont sélectionnés par un discriminateur d'amplitude et comptés par périodes d'1 s. Les effets des substances appliquées sont évalués par les variations de la fréquence des potentiels d'action enregistrée graphiquement. Les trois canaux restants contiennent respectivement : une solution de $\mathrm{NaCl} 2 \mathrm{M}$ permettant de contrebalancer les effets des courants appliqués pour libérer les substances actives, une solution de glutamate (Sigma, IM) et une solution de prolactine humaine (Calbiochem, $2 \mu$ g dans 50 ou $100 \mu \mathrm{l}$ de $\mathrm{NaCl} 0,9$ p. 100). Les substances sont libérées au voisinage de la membrane cellulaire par des courants continus de polarité adaptée.

Après chaque expérience, le cerveau de l'animal est fixé et des coupes à congélation de $80 \mu$ sont effectuées dans les régions étudiées pour contrôler les trajets des électrodes.

\section{Résultats.}

L'effet stimulant du glutamate appliqué par des courants négatifs de 10 à $50 \mathrm{nA}$ a permis dans chaque cas de s'assurer de la position de la microélectrode auprès d'un péricaryon et de rendre possible l'étude des effets inhibiteurs de la PRL sur des neurones présentant une activité spontanée faible ou inexistante.

63 neurones ont été analysés dans le septum et l'APO. 18 neurones ont été reconnus activés antidromiquement par la stimulation de l'éminence médiane après avoir satisfait à l'essai de la collision. Dans ce cas le potentiel d'action évoqué par la stimulation est annulé lors de l'apparition d'un potentiel d'action spontané. De plus le potentiel d'action antidromique est recueilli avec une latence constante. Un épaulement dans la phase montante de ce potentiel d'action divise parfois celui-ci en deux composantes : une composante $A$ à latence fixe et une composante $B$ à latence variable. Après stimulation à haute fréquence ou application de deux chocs successifs, on observe une séparation plus nette de ces deux composantes s'accompagnant d'une inhibition de la dernière.

Parmi les 63 cellules soumises à la PRL, libérée par des courants négatifs de 10 à $50 \mathrm{nA}$ appliqués pendant des périodes de 5 à $30 \mathrm{~s}, 27$ ne montrèrent aucune modification de leur fréquence d'activité, 6 furent inhibées ef 30 activées.

1) Cellules inhibées par la prolactine.

Pour 6 cellules, la fréquence des potentiels d'action spontanés ou déclenchés par le glutamate est inhibée lors de l'application micro-iontophorétique de PRL. Cette 
inhibition est limitée au temps d'application de la substance. Elle apparaît entre 0,5 et $2 \mathrm{~s}$ après la libération de PRL par la microélectrode et le niveau d'activité initial du neurone est rétabli quelques secondes après l'application. La latence et la durée de l'effet, la stabilité de la réponse vis-à-vis des applications répétées, la relation proportionnelle entre l'intensité des courants d'application et la réponse enregistrée satisfont aux critères de Curtis et Koizumi (1961) concernant l'application de neurotransmetteurs par micro-iontophorèse et éliminent l'hypothèse d'une action directe du courant polarisant sur la membrane cellulaire.

\section{2) Cellules activées par la prolactine.}

Pour 30 cellules, la fréquence des potentiels d'action spontanés est augmentée ou une activité prend naissance dans un neurone préalablement silencieux lors de l'application micro-iontophorétique de PRL.

L'action de la substance se manifeste ici de deux manières différentes. Dans un premier cas, c'est une activation limitée au temps d'application de la PRL qui se manifeste dans 3 cellules, avec un délai et un retour à l'état de base semblables à ceux décrits pour les cas d'inhibition. Dans un second cas, c'est une activation importante ef prolongée, dépassant le temps d'application de la PRL qui est enregistrée chez 27 cellules. Cet effet activateur débute pendant l'application de l'hormone mais peut naître également après que la PRL ait éfé libérée. Le temps mis pour retrouver le niveau d'activité initial varie dans de fortes proportions et la cellule peut ne pas retrouver ce niveau d'activité pendant la période d'enregistrement stable, ce qui ne permet pas d'établir une moyenne du temps d'action. Enfin on remarque dans ce second cas une augmentation consistante de l'effet avec des applications répétées de PRL. On peut constater également que ce type d'activation avec effets prolongés est semblable à celui obtenu dans les mêmes régions par application micro-iontophorétique de prostaglandines $\mathrm{PGE}_{2}$ et $\mathrm{PGF}_{2 \alpha}$ (Poulain et Carette, 1974).

Les 63 cellules soumises à l'application de PRL se répartissent entre les plans 12,8 et 13,4 de notre atlas stéréotaxique (Poulain, 1974), dans la région périventriculaire (aire préoptique médiane), les noyaux des bandelettes diagonales de Broca et du septum et se distribuent latéralement sur $0,8 \mathrm{~mm}$ à partir du bord du ventricule. La répartition des neurones étudiés selon leur type de réponse est indiquée dans le tableau suivant :

\begin{tabular}{|c|c|c|c|c|c|}
\hline & \multicolumn{2}{|c|}{ SEPTUM } & \multicolumn{2}{|c|}{ APO } & \multirow{2}{*}{$\begin{array}{c}\text { Nombre } \\
\text { total } \\
\text { de } \\
\text { cellules }\end{array}$} \\
\hline & $\begin{array}{c}\text { Activité } \\
\text { antidromique }\end{array}$ & $\begin{array}{c}\text { Activité } \\
\text { spontanée }\end{array}$ & $\begin{array}{c}\text { Activité } \\
\text { antidromique }\end{array}$ & $\begin{array}{l}\text { Activité } \\
\text { spontanée }\end{array}$ & \\
\hline Pas d'action......... & 6 & 11 & 3 & 7 & 27 \\
\hline Inhibition & 0 & 3 & 1 & 2 & 6 \\
\hline $\begin{array}{l}\text { Activation } \\
\mathbf{1}^{\text {er type }} . \ldots \ldots \ldots \ldots \\
2^{\mathrm{e}} \text { type } . . \ldots \ldots \ldots\end{array}$ & $\begin{array}{l}0 \\
4\end{array}$ & $\begin{array}{l}1 \\
3\end{array}$ & $\begin{array}{l}0 \\
4\end{array}$ & $\begin{array}{r}2 \\
16\end{array}$ & $\begin{array}{r}3 \\
27\end{array}$ \\
\hline
\end{tabular}




\section{Discussion. Conclusions.}

Deux travaux récents ont démontré que l'application micro-iontophorétique de PRL sur les neurones diencéphaliques se traduisait en majorité par une activation de leur activité unitaire. Cependant chez le Rat mâle, Yamada (1975) n'observe pas d'effet de la substance dans la région préoptique, tandis que Kawakami et Sakuma (1976) chez la femelle notent des effets localisés uniquement aux neurones de l'aire périventriculaire activés antidromiquement par la stimulation de l'hypothalamus médio-basal. Ce travail réalisé chez le Cobaye apporte les trois constatations suivantes : action activatrice en majorifé de la PRL, existence de neurones sensibles à cette hormone dans les régions préoptiques et septales, action non limitée aux cellules ayant un rapport direct avec l'éminence médiane. Enfin est décrit un type d'activation particulier avec effets prolongés se manifestant plutôt au niveau des neurones de l'APO.

Il est impossible de définir actuellement le rôle physiologique des neurones sensibles à la PRL, cependant il est possible que certains d'entre eux participentà la régulation de la sécrétion de l'hormone en étant impliqués dans un mécanisme de rétroaction courte de celle-ci.

$8 \mathrm{e}$ Colloque de Neuroendocrinologie. - Table ronde Prolactine. Tours, 7 septembre 1976.

\section{Références}

CLEMENS J. A., GALLO R. V., WHITMOYER D. I., 1971a. Prolactin responsive neurons in the rabbit hypothalamus. Brain Res., 25, 371-379.

CLEMENS J. A., SHAAR C. J., KLEBER J. W., TANDY W. A., 1971b. Reciprocal control by the preoptic area of LH and prolactin. Exp. Brain Res., 12, 250-253.

CURTIS D. R., KOIZUMI K., 1961. Chemical transmitter substances in brain stem of cat. J. Neurophysiol. 24, 80-90.

KAWAKAMI M., KIMURA F., KONNO T., 1973a. Possible role of the medial prechiasmatic area in the release of LH and prolactin in rats. Endocr. jap., 20, 335-344.

KAWAKAMI M., SAKUMA Y., 1976. Electrophysiological evidences for possible participation of periventricular neurons in anterior pituitary regulation. Brain Res., 101, 79-94.

KAWAKAMI M., TERASAWA E., KIMURA F., WAKABAYASHI K., 1973b. Modulatin effect of limbic structures on gonadotropin release. Neuroendocrinology, 12, 1-16.

MALVEN P. V., 1975. Prolactin release induced by electrical stimulation of the hypothalamic preoptic area in unanesthetized sheep. Neuroendccrinology, 18, 65-71.

POULAIN P., 1974. L'hypothalamus et le septum du cobaye de $400 \mathrm{~g}$ en coordonnées stéréotaxiques. Arch. Anat. micr. Morph. exp., 63, 37-50.

POULAIN P., CARETTE B., 1974. Iontophoresis of prostaglandins on hypothalamic neurons, Brain Res., 79, 311-314.

POULAIN P., PARTOUCHE C., 1973. Relations des neurones de la région préoptique et du septum avec le noyau arqué ef l'éminence médiane. C. R. Acad. Sci. Paris, Série D, 277, 737-739.

WUTTKE W., MEITES J., 1972. Effects of electrochemical stimulation of the hypothalamus on serum prolactin and LH in rats. Pflüg. Arch. ges. Physiol., 337, 71-79.

YAMADA Y., 1975. Effects of iontophorefically applied prolactin on unit activity of the rat brain. Neuroendocrinology, 18, 263-271. 